\title{
Monte Bajo, opción para tres especies de Eucaliptos en segunda rotación, un caso en la provincia de Osorno, Décima Región, Chile
}

\author{
Coppice options for three Eucalyptus species, a case in the Osorno Province, X Region \\ EDITH GELDRES, ${ }^{1}$ JUAN E. SCHLATTER,${ }^{2}$ ANDRES MARCOLETA ${ }^{1}$ \\ ${ }^{1}$ Departamento de Ciencia y Tecnología Forestal, Universidad de Los Lagos, Casilla 933, Osorno, Chile. \\ E-mail: edgeldres@surnet.cl \\ ${ }^{2}$ Instituto de Silvicultura, Facultad de Ciencias Forestales, Universidad Austral de Chile, Casilla 567, \\ Valdivia, Chile. E-mail: jschlatt@uach.cl
}

\section{SUMMARY}

In the $\mathrm{X}$ Region large eucalyptus plantations have been established, and these are currently harvestable. The growers will now have to decide between the alternatives of either establishing a new forestry crop or managing eucalyptus under a coppice regime. Notable amongst those species that have the ability to sprout and form low forest are Eucalyptus globulus and E. viminalis. A management trial was carried out for a 8.0 ha coppice of mixed E. globulus, E. viminalis, and E. regnans, harvested at eight years of age. The trial was located near Huilma, Province of Osorno, where the soil is of the Crucero series (red clayey soil), and this was evaluated four years after the harvest. The species showing a possibility of management under a coppice regime were E. globulus and E. viminalis. The first of these has a better volume production: $150 \mathrm{~m}^{3} / \mathrm{ha}$ in four years. If the intended purpose is use as pulp or firewood, it is recommended that three sprouts per stump are left 18 months after harvesting. However, if the use is as sawn wood, then both species need to be managed with one or two sprouts per stump.

Key words: coppice, eucalyptus, red clayey soils.

\section{RESUMEN}

En la Décima Región de Los Lagos se han establecido extensas plantaciones de eucaliptos que están siendo cosechadas o lo serán muy pronto. Los propietarios deberán decidir entre dos alternativas: si volver a establecer un nuevo cultivo forestal o manejar los eucaliptos bajo régimen de monte bajo. Entre las especies que tienen la capacidad de rebrotar y formar un bosque de monte bajo destacan Eucalyptus globulus y Eucalyptus viminalis. Por este motivo se establecieron ensayos de manejo como monte bajo en una plantación mixta de Eucalyptus globulus, Eucalyptus viminalis y Eucalyptus regnans, de 8 ha y cosechada a los 8 años de edad. El ensayo se ubica cerca de Huilma, provincia Osorno, donde el suelo corresponde a la serie Crucero (rojo arcilloso) y fue evaluado a los 4 años después de la cosecha. Las especies que demostraron posibilidad de ser manejadas como monte bajo son: Eucalyptus globulus y Eucalyptus viminalis. Entre ellas la primera muestra mayor vigorosidad, especialmente para la producción de volumen ( $150 \mathrm{~m}^{3} / \mathrm{ha}$ en 4 años), si el objetivo es madera para pulpa o leña, recomendándose en ese caso dejar tres brotes por tocón después de 18 meses desde la cosecha. Sin embargo, también se presenta como una posibilidad el producir madera para aserrar, en estos casos ambas especies deben ser manejadas con uno a dos brotes por tocón.

Palabras clave: monte bajo, eucaliptos, suelos rojo arcillosos. 
BOSQUE 25(3): 57-62, 2004

Monte Bajo, opción para tres especies de Eucaliptos en segunda rotación, un caso en la provincia de Osorno...

\section{INTRODUCCION}

En Chile es notorio el mayor interés, por parte de las empresas y los propietarios particulares, en la creación de bosques de eucaliptos para la producción de: leña, pulpa, tableros, chapas y madera aserrada, además de productos secundarios como aceites esenciales, taninos y otros. En la región de Los Lagos (Décima Región) se han establecido extensas plantaciones de eucaliptos, de las cuales un gran porcentaje está entrando al ciclo de corta. Esto exige al propietario la definición de cuál alternativa seleccionar, establecer una nueva plantación o aprovechar la capacidad de rebrote de los eucaliptos. En el caso de esta última alternativa debe definir qué esquema de manejo seguirá para obtener el mejor resultado.

Algunos eucaliptos tienen la facultad de brotar vigorosamente de tocón, destacando entre ellos Eucalyptus globulus, el cual puede producir biomasa en cantidades considerables a través del método silvícola de "monte bajo". El término "monte bajo" se aplica a los bosques que han regenerado a partir de brotes de tocón, dejados por la cosecha de un monte alto, y se emplea con éxito en un amplio grupo de especies del género Eucalyptus (1).

Sin embargo, no todas las especies de eucaliptos son adecuadas para el manejo como monte bajo, ya sea porque su capacidad de brotación es pobre o porque los retoños producidos no logran una vigorosidad suficiente para obtener madera de dimensiones comerciales. Es decir, cuando no se puede contar con la regeneración por monte bajo debe procederse necesariamente a la reforestación. Entre las especies de eucaliptos con una pobre capacidad de rebrotar pueden mencionarse Eucalyptus fastigata, Eucalyptus nitens y Eucalyptus regnans, entre otras (2).

En Chile, las plantaciones de eucaliptos manejados con el sistema de monte bajo son especialmente difundidas en la zona Centro-Norte (V-VIII Regiones), donde se les utiliza para obtener madera de cortas dimensiones (3). Según este autor, los rendimientos de monte bajo son muy variables a través de las sucesivas rotaciones, las que se efectúan sobre los mismos tocones, imposibilitando prever un rendimiento constante de la masa. Sin embargo, las dos primeras rotaciones en este sistema son en general muy productivas.
En la Décima Región, la información de manejo de plantaciones de eucaliptos, como monte bajo, prácticamente no existe. Por este motivo se desarrolló el presente estudio, que tiene como objetivo contribuir al tema por medio de la evaluación del crecimiento de tres especies de eucaliptos bajo régimen de monte bajo, en segunda rotación.

\section{MATERIAL Y METODOS}

Las plantaciones analizadas están ubicadas en el sector de Huilma, situado a $20 \mathrm{~km}$ al sur de la ciudad de Osorno, comuna de Río Negro, Décima Región. El bosque estudiado tiene una superficie de 8 ha, de composición mixta y conformado por Eucalyptus globulus (242 árboles/ha), Eucalyptus viminalis (608 árboles/ha) y Eucalytptus regnans (200 árboles/ha). Fue establecido en el año 1989 con plantas a raíz desnuda, con un mínimo control de malezas: sólo a nivel de taza, y sin fertilización. A los 8 años de edad se cosecha a tala rasa y luego se espera un período de 18 meses para que los tocones rebroten y desarrollen los retoños, definiéndose en ese período los pies más vigorosos. En enero del año 1999 se inician los ensayos de manejo silvícola, como también las mediciones.

El área de crecimiento se caracteriza por un clima templado-cálido con menos de 4 meses secos (4), con precipitaciones de 1.400-1.600 mm anuales y una temperatura media anual de alrededor de $11^{\circ} \mathrm{C}$. La temperatura media mensual más alta es en enero con $16,4^{\circ} \mathrm{C}$ y la menor en julio con $6,8^{\circ} \mathrm{C}$. El período libre de heladas fluctúa entre 150-200 días, entre octubre y abril, con una frecuencia de 20-30 heladas al año, las que pueden alcanzar los $-6^{\circ} \mathrm{C}$, pero con una frecuencia no más allá que durante un mes (5).

Los suelos del sitio corresponden a la serie Crucero, rojo arcillosos generalmente muy profundos, que yacen sobre conglomerados volcánicos muy meteorizados. Las texturas dominantes son arcillosas, pero por su adecuada estructura presentan un buen drenaje. En el horizonte A, de $10-16 \mathrm{~cm}$ de profundidad, presentan un alto nivel de materia orgánica (6).

En la plantación de eucaliptos mencionada se establece un diseño de bloques al azar con tres repeticiones, considerando como unidad experi- 
BOSQUE 25(3): 57-62, 2004 Monte Bajo, opción para tres especies de Eucaliptos en segunda rotación, un caso en la provincia de Osorno...

mental parcelas de $100 \mathrm{~m}^{2}$, los tratamientos establecidos en los ensayos fueron: selección de 1 retoño por tocón (tratamiento 1: T1); selección de 2 retoños por tocón (Tratamiento 2: T2); selección de 3 retoños por tocón (Tratamiento 3: T3); sin intervención o situación testigo (Tratamiento 4: T4).

Criterios de manejo para el establecimiento de los tratamientos: el manejo se ejecutó dejando aquellos retoños que se encontraban fuertemente adheridos al tocón y además prefiriendo a los que se encontraban en la cara del tocón orientada a favor del viento dominante. Se eliminaron los retoños que presentaban doble flecha, bifurcación y curvatura en el fuste. Se prefirió dejar brotes proventicios, en vez de adventicios (1). En la situación de presentarse dos retoños en un mismo callo, se eliminó uno de ellos, para facilitar el crecimiento del retoño a dejar.

Medición de las variables de cada tratamiento: las variables medidas en cada uno de los tratamientos fueron: el diámetro de tocón, el diámetro a la altura de pecho (DAP) y la altura total. La medición de alturas en los dos primeros años se realizó con una vara graduada en centímetros, en cambio, para el tercer y cuarto año se utilizó hipsómetro. La medición de diámetros se realizó con pie de metro los dos primeros años, y el tercer y cuarto año se utilizó forcípula. Los ensayos se han evaluado durante 4 años, mediciones que fueron iniciadas a fines de enero de cada año, desde 1999.

Para la estimación de volumen de los tocones se utilizó la siguiente expresión:

\section{$\mathrm{V}=0,00003003391 * \mathrm{DAP}^{2} * \mathrm{~h}$}

Ribalta (1983) ${ }^{1}$ cit. por Prado y Barros (1).

El volumen es el volumen cúbico total en el fuste $\left(\mathrm{m}^{3}-\mathrm{ssc}\right)$.

La evaluación de vigorosidad de las especies de E. globulus, E. viminalis y E. regnans fue efectuada sin aplicación de fertilizantes ni control de malezas y tampoco se modificaron las características físicas del suelo.

1 RIBALTA, E. Evaluación de la producción y productividad monte bajo de Eucalyptus globulus (Lab), V Región. Tesis. Escuela de Ingeniería Forestal, Universidad de Chile. Santiago. 124 p.

\section{RESULTADOS}

Los resultados de vigorosidad de los retoños de las distintas especies de Eucalyptus sp. asociadas en una plantación mixta de 8 años, se observan en las figuras 1, 2 y 3 , respectivamente. En un período de 4 años fueron comparadas las tres especies a través del tratamiento T1 (un retoño por tocón). Se evalúan individualmente $E$. globulus, E. viminalis y E. regnans.

En la figura 1 destacan E. globulus y $E$. viminalis como las especies de rebrote más vigoroso; en cambio, E. regnans muestra un desarrollo mucho más débil. E. globulus es la especie de mayor vigor en crecimiento en altura.

La figura 2 muestra similar tendencia en el diámetro (DAP), observándose el mayor vigor en E. globulus, seguido de cerca por E. viminalis,

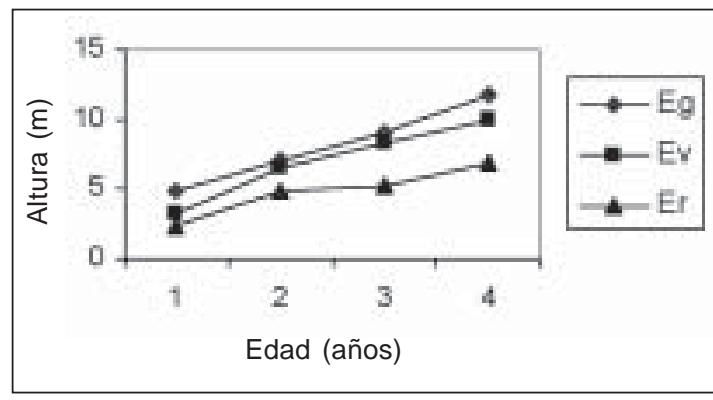

Figura 1. Altura promedio de Eucalyptus sp. a distintas edades, como monte bajo (un retoño por tocón), serie Crucero, provincia de Osorno, Décima Región. Average height for different ages of Eucalyptus sp. as mixed coppice forest (one sprout per stump), Crucero soil series, province of Osorno, X Region.

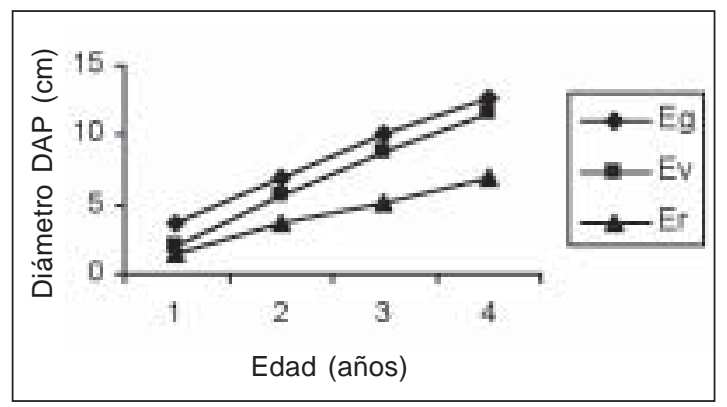

Figura 2. Diámetro promedio de Eucalyptus sp. a diferentes edades, en monte bajo (un retoño por tocón), serie Crucero, provincia de Osorno, Décima Región.

Average diameter for different ages of Eucalyptus sp. as mixed coppice forest (one sprout per stump), Crucero soil series, province of Osorno, X Region. 


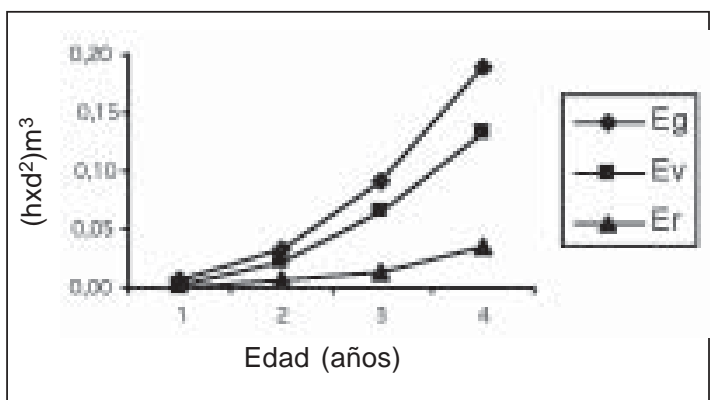

Figura 3. Indice de productividad para de Eucalyptus sp., como monte bajo mixto (un retoño por tocón), serie Crucero, provincia de Osorno, Décima Región. Production index for Eucalyptus sp. as mixed coppice forest (one sprout per stump), Crucero soil series, province of Osorno, X Region.

E. regnans nuevamente muestra un desarrollo menor.

En la figura 3 se presenta el desarrollo de los eucaliptos expresado como índice de productividad $\left(\mathrm{DAP}^{2 *} \mathrm{~h}\right)$, el cual como integrador de las variables de crecimiento anteriores da como resultado un rendimiento mayor en E. globulus. E. viminalis muestra una buena vigorosidad pero de menores dimensiones que E. globulus. E. regnans, en cambio, presenta un desarrollo limitado.

De lo anterior se desprende que la especie que mejores proyecciones tiene en el sitio en cuestión es E. globulus y en segundo lugar E. viminalis, considerando que las plantaciones se desean manejar como monte bajo. Sin embargo, aún debe evaluarse cuál es el sistema silvícola más conveniente para el sitio, tanto desde el punto de vista biológico como también financiero.

Los distintos tratamientos silvícolas se evaluaron en sus resultados por intermedio de una comparación del rendimiento al año 4, entre las tres especies, considerando para este efecto el promedio de las tres repeticiones. Estos resultados se entregan en las figuras 4, 5 y 6.

En figura 4 puede observarse la reacción, en altura promedio, que tuvieron las diferentes especies para distinto número de retoños por tocón. El mayor vigor de E. globulus se observa en todas las densidades de rebrote consideradas. En cambio E. viminalis reacciona positivamente sólo hasta con dos retoños, después tiende a decaer. Algo similar se observa en E. regnans. La mejor altura la logró E. globulus con tres retoños.

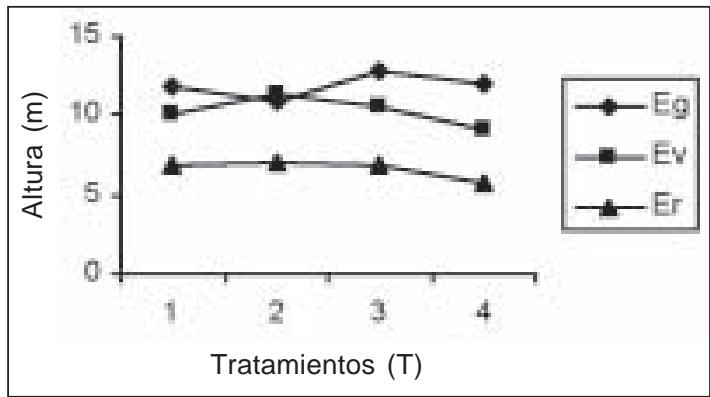

Figura 4. Crecimiento promedio en altura en rebrotes de Eucalyptus sp., como monte bajo mixto, serie Crucero, provincia de Osorno, Décima Región.

Average height growth of Eucalyptus sp. as mixed coppice forest, Crucero soil series, province of Osorno, X Region.

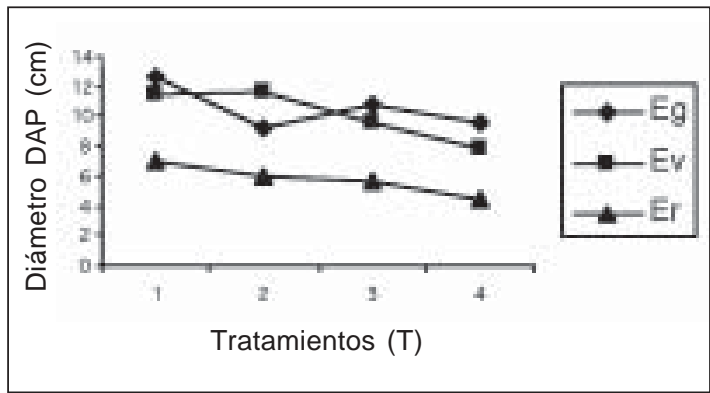

Figura 5. Diámetro promedio en rebrotes de Eucalyptus sp., como rodal mixto, serie Crucero, provincia de Osorno, Décima Región.

Average diameter of Eucalyptus sp. as mixed coppice forest, Crucero soil series, province of Osorno, X Region.

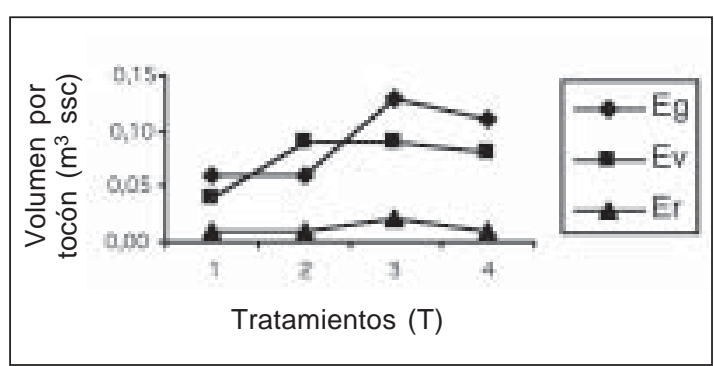

Figura 6. Volumen ( $\mathrm{m}^{3}$ (ssc)/tocón) en Eucaliptus sp., como rodal mixto, serie Crucero, provincia de Osorno, Décima Región.

Volume of production ( $\mathrm{m}^{3}$ u.b./stump) of Eucalyptus sp. as mixed coppice forest, Crucero soil series, province of Osorno, $\mathrm{X}$ Region.

La tendencia de aumentar la altura del $E$. globulus, desde uno a tres retoños, puede explicarse por la mayor competencia por luz al aumentar la densidad de estos por tocón. Sin embargo, 
BOSQUE 25(3): 57-62, 2004

Monte Bajo, opción para tres especies de Eucaliptos en segunda rotación, un caso en la provincia de Osorno...

más de tres retoños acusan un rendimiento decreciente. En efecto, la situación testigo (T4), con más de 4 retoños por tocón, mostró una condición de estrés desde el año 1.

En la figura 5 se presentan los resultados obtenidos por retoños de Eucalyptus sp., en el diámetro (DAP). Para las tres especies el tratamiento T1 (un retoño por tocón) es el que obtiene la mayor dimensión en el diámetro promedio, alcanzando su máximo valor con $12,7 \mathrm{~cm}$ para $E$. globulus. Esta mayor vigorosidad en diámetro se explica por la menor competencia al existir menos retoños, lo que significa que a una misma edad de rotación este tratamiento obtendrá un producto de mayores dimensiones comerciales.

En la figura 6 se muestran los volúmenes (sin corteza) por tocón, que logran las distintas especies de eucaliptos. E. globulus y E. viminalis muestran resultados muy similares hasta una densidad de dos retoños por tocón, pero "globulus" supera a esta última especie en los tratamientos con tres o más retoños. E. regnans, en cambio, observa una reacción débil. El resultado del volumen promedio con dos retoños en E. globulus escapa de lo que se podría esperar. Este hecho se explica por los daños causados por viento a los retoños más vigorosos en las parcelas de este tratamiento.

De acuerdo a las tendencias de vigorosidad de las 3 especies de eucaliptos estudiadas, se deduce que es factible manejar E. globulus originado por rebrote como monte bajo en la segunda rotación. La especie $E$. viminalis también expresó una buena capacidad de retoñación, pero logrando menores dimensiones. E. regnans, en cambio, no es una buena alternativa para un manejo tipo monte bajo. Esta última especie tiene un buen potencial en la Décima Región, pero para ser cultivada en plantaciones en régimen de monte alto.

Entre las tres especies de eucaliptos analizadas en su rendimiento bajo el sistema de "monte bajo" se pudo distinguir a E. globulus como la de mayor vigor en los rebrotes. E. viminalis también muestra un vigor interesante cuando es manejada con uno y especialmente dos retoños por tocón. Sin embargo, "globulus" la supera al aumentar el número de retoños, presentando los mejores rendimientos con tres retoños al lograr $0,13 \mathrm{~m}^{3} /$ tocón, lo que significa un volumen de producción finalmente mayor. Si se proyecta a una hectárea la capacidad de producción de esta especie, ésta fluctuaría alrededor de $\operatorname{los} 150 \mathrm{~m}^{3} / \mathrm{ha}$ para una densidad de 1.200 árboles/ha. Esto supera la capacidad de producción volumétrica de la primera rotación como monte alto, para igual período (6), lo que indica que es una buena alternativa para proyectar este tipo de manejo en la especie, sea cual fuere el tipo de propietario, especialmente cuando el objetivo es madera pulpable o leña (bioenergía). Aún mejores rendimientos podrían obtenerse en el caso que se efectuara control de malezas y se tomaran medidas de manejo nutritivo (fertilización), medidas que no fueron aplicadas en las plantaciones estudiadas.

Si el objetivo del manejo de la segunda rotación de monte bajo es obtener madera con dimensiones aserrables o debobinables es recomendable dejar entre uno y dos retoños por tocón en las dos mejores especies. En cambio, si el objetivo de la plantación es la producción de volumen para un uso pulpable o bioenergético, dejar 3 retoños por tocón es una mejor alternativa de manejo en $E$. globulus.

El resultado aquí logrado contribuye al esclarecimiento de las posibilidades de los eucaliptos en la región y no cabe duda que la alternativa de rebrote en E. globulus favorece a esta especie como cultivo forestal.

\section{CONCLUSIONES}

El mayor rendimiento como monte bajo en la segunda rotación lo presenta $E$. globulus. En cambio, E. regnans es la especie que registró el menor vigor, situación que confirma su mala capacidad de rebrote, debiéndose descartar para una producción como monte bajo.

El mayor vigor lo presenta E. globulus, que supera a E. viminalis. Esto se deduce tanto de los resultados en diámetro, como en altura, ya que ambas variables son mayores en la primera especie, especialmente con más de dos retoños por tocón.

E. globulus logra el mayor volumen de producción en todos los tratamientos pero el óptimo lo obtiene con tres retoños por tocón. En cambio, E. viminalis muestra resultados que indican que para ella el óptimo es manejarla con no más de dos retoños. Esto responde bien al objetivo de producción para pulpa o leña. 
BOSQUE 25(3): 57-62, 2004

Monte Bajo, opción para tres especies de Eucaliptos en segunda rotación, un caso en la provincia de Osorno...

Sin embargo, si el objetivo de la segunda rotación de monte bajo de E. globulus o E. viminalis es obtener un producto de dimensiones comerciales para aserrar, se recomienda dejar uno o dos retoños por tocón.

Los resultados de manejo de monte bajo en $E$. globulus a los 4 años de edad permiten proyecciones interesantes, dado que registran crecimientos en diámetro, altura y volumen mayores a los valores obtenidos en plantaciones de la misma especie y en el mismo período con un régimen de monte alto.

\section{BIBLIOGRAFIA}

(1) PRADO, J.A., S. BARROS. Eucalyptus. Principios de silvicultura y manejo. Santiago de Chile: Instituto Forestal, División Silvicultura, CORFO. 1989. 199 p.
(2) FAO. El Eucalipto en la repoblación forestal. Colección Montes $\mathrm{N}^{\circ} 11$. Food and Agriculture Organization, Naciones Unidas, Roma. 1981. 723 p.

(3) CAMERATTI, A. Estudio de la brotación de tocones de Eucalyptus globulus (lab), Valdivia. Tesis. Universidad Austral de Chile, Facultad de Ingeniería Forestal. 1969. $37 \mathrm{p}$.

(4) FUENZALIDA, H. Clima. Geografía Económica de Chile, Corporación de Fomento de la Producción. 1965, p. 99-152.

(5) SCHLATTER, J. E., V. GERDING, H. HUBER. Sistema de ordenamiento de la Tierra. Herramienta para la planificación forestal, aplicado a la Décima Región. Serie Técnica, Fac. Cs. Forestales, Univ. Austral de Chile, Valdivia, Chile. 1995. 93 p.

(6) TOSSO, J. Suelos volcánicos de Chile. Santiago: Ed Instituto de Investigaciones Agropecuarias (INIA), Ministerio de Agricultura, Chile. 1985. 723 p.

(7) GELDRES, E., J. E. SCHLATTER. Crecimiento de las plantaciones de Eucalyptus globulus sobre suelos rojo arcillosos de la provincia de Osorno, Décima Región. Bosque (Chile), 2004. vol. 25, No 1, (en prensa). 